\title{
Sylvia H. Heywang-Koebrunner, Ingrid Schreer and Susan Barter (Eds): Diagnostic Breast Imaging: Mammography, Sonography, Magnetic Resonance Imaging and Interventional Procedures, 3rd edn
}

\author{
Georg Thieme Verlag, Stuttgart, 2014; ISBN 978-3-13-1028938
}

\author{
Vincenzo Cuccurullo • Antonio Santoriello
}

Published online: 28 August 2014

(C) Springer-Verlag Berlin Heidelberg 2014

Diagnostic breast imaging has a proven role in the early detection of breast cancer and can significantly contribute to improving the duration and quality of life of women. This book is the third updated edition of a successful text written by a team led by Sylvia H. Heywang-Koebrunner, Professor and Head of the Reference Center for Mammography in Munich, and also including as coeditors Ingrid Schreer, Professor at the Radiologic Center in Hamburg, and Susan Barter, Consultant Radiologist at the Breast Unit of Cambridge's University, UK. The contributions of Fleur Kilburn-Toppin, John Kotre and Joerge Naehrig are also recognized.

This new edition of 696 pages, enriched by 1,205 illustrations, is divided into three sections ("Methods", "Appearance", and "AApplication of Diagnostic Imaging of the Breast") organized into 24 comprehensive chapters. The first section (chapters 1-8) starts with a brief analysis of the relevance of patient history, communication with the patient and clinical findings as a mandatory premise to clinical diagnostic imaging. Major techniques (mammography, sonography and magnetic resonance imaging) are then comprehensively described in depth, followed by updated but concise information on innovative and/ or less diffuse techniques, including nuclear medicine. The section concludes with the most important information concerning percutaneous biopsy and preoperative localization.

The second section (chapters 9-22), the most extensive, starts with the study of the normal breast and then analyses all the

V. Cuccurullo $\cdot$ A. Santoriello

Second University of Naples, Naples, Italy

V. Cuccurullo $(\bowtie)$

Medicina Nucleare, Seconda Università di Napoli, P.zza Miraglia,

2-80138 Napoli, Italy

e-mail: vincenzo.cuccurullo@unina2.it possible pathological conditions, also considering lymph node involvement and less frequent pathologies, such as those involving the male breast. The chapters are structured to include all the most important information, starting from anatomy, histology, aetiology, clinics and so on, to arrive at the best interpretation of images acquired with different techniques. The clinical role of the various imaging procedures is discussed analysing achievable information, indications, possibilities and limitations so that the cost-effectiveness in an individual patient can be defined based on the highest accuracy and/or the lowest cost. All chapters also include updated references and recommended reading. The third section (Application of Diagnostic Imaging of the Breast) includes chapter 22 on screening and chapter 23 describing additional diagnostic evaluation of screening findings and the solving of problems in symptomatic patients.

This book has been fully updated to include advances in mammography, ultrasonography, breast MRI, interventional procedures and emerging technologies. Special features of this highly regarded edition are the more than 1,200 radiographic images showing normal findings, benign and malignant disorders, posttraumatic, postsurgical, and posttherapeutic changes in the breast. Furthermore, innovations in digital mammography, tomosynthesis, computer-assisted detection (CAD), positron emission tomography and scintimammography are also included, and there are in addition new chapters on imaging of implants and lesions of uncertain malignant potential, also analysed using new developing technologies and/or innovative procedures.

This book is strongly recommended to all those interested in breast imaging, including not only clinical radiologists, but also practitioners, oncologists, gynaecologists, surgeons and students. In general, this publication can be considered a reliable and useful guide for those who want to learn breast diagnostics in its clinical context. 